\title{
Temporal changes in brachyuran crab diversity along heterogeneous habitat in a mangrove ecosystem of Indian Sundarbans
}

\author{
Shilpa Sen, Sudeshna Mukherjee, Atreyee Chaudhuri, Sumit Homechaudhuri \\ Aquatic Bioresource Research Laboratory, Department of Zoology, University of Calcutta, 35 Ballygunge Circular Road, \\ Kolkata 700019, India. E-mail: sumithomec@yahoo.com
}

\begin{abstract}
Summary: The present study investigates the effect of different habitat attributes on brachyuran crab diversity in two different study sites in the Sundarban mangrove, India. The two sites differ in the level of anthropogenic intrusion and in the age of the mangrove forest. Seasonal changes in the environment and in brachyuran faunal abundance were recorded for three years. Species composition varied between the two habitats irrespective of season. The habitat heterogeneity and the recorded crab community was analysed by several univariate and multivariate statistical techniques. The newly replanted mangrove site showed lesser diversity than the natural one. Ocypodid crabs, mainly Uca rosea, dominated both study sites, whereas Uca triangularis was totally absent from the replanted site. Canonical correspondence analysis showed that the total acidity, total alkalinity, $\mathrm{pH}$ content of water, total dissolved solids, inorganic phosphate content of water, soil specific gravity, soil density and the physical constructions of the habitat play a crucial role in moderating the crab community structure. This study reveals that brachyuran crab diversity can be used as a potential indicator of the alterations of mangrove habitats.
\end{abstract}

Keywords: crab diversity; canonical correspondence analysis; edaphic qualities; habitat heterogeneity; hydrological parameters; mangroves.

Cambios temporales en la diversidad de los cangrejos braquiuros a lo largo de un hábitat heterogéneo del manglar indio de Sundarban

Resumen: El presente estudio investiga el efecto de las diferentes características de hábitat sobre la diversidad de los cangrejos braquiuros en dos lugares diferentes del manglar Sundarban, India. Los dos sitios difieren en el nivel de intrusión antropogénica, así como en la edad del bosque de manglar. Se registraron cambios estacionales en el medio y en la abundancia de la fauna de braquiuros durante tres años. A pesar de las diferencias estacionales, la composición de especies difiere en los dos hábitats. Se analizó la heterogeneidad del hábitat y la comunidad de cangrejos mediante técnicas estadísticas univariantes y multivariantes. La zona de manglares replantada recientemente mostró menor diversidad que la zona natural. Los cangrejos ocipódidos, principalmente Uca rosea, dominaron en ambas zonas de estudio, mientras que Uca triangularis estuvo totalmente ausente en la zona replantada. El análisis de Correspondencia Canónica (CCA) mostró que la acidez total, la alcalinidad total, el contenido de $\mathrm{pH}$ del agua, los sólidos totales disueltos (TDS), el contenido de fosfato inorgánico del agua, el peso específico del suelo, la densidad del suelo, junto con las construcciones físicas del hábitat desempeñan un papel fundamental en la estructura de la comunidad de cangrejos. Este estudio revela que la diversidad de los cangrejos braquiuros puede ser utilizada como un potencial indicador de las alteraciones de los hábitats de manglares.

Palabras clave: diversidad de cangrejos; análisis de correspondencia canónica; cualidades edáficas; heterogeneidad del hábitat; parámetros hidrológicos; manglares.

Citation/Como citar este artículo: Sen S., Mukherjee S., Chaudhuri A., Homechaudhuri S. 2014. Temporal changes in brachyuran crab diversity along heterogeneous habitat in a mangrove ecosystem of Indian Sundarbans. Sci. Mar. 78(3): 433442. doi: http://dx.doi.org/10.3989/scimar.03931.04A

Editor: J.A. Cuesta.

Received: August 02, 2013. Accepted: June 06, 2014. Published: August 4, 2014.

Copyright: (c) 2014 CSIC. This is an open-access article distributed under the Creative Commons Attribution-Non Commercial Lisence (by-nc) Spain 3.0. 


\section{INTRODUCTION}

Mangroves are the most productive and dynamic ecosystems in tropical and subtropical intertidal areas (Robertson and Blaber 1992, Kathiresan and Bingham $2001)$. The Sundarban mangrove $\left(21^{\circ} 30^{\prime}\right.$ to $22^{\circ} 40^{\prime} \mathrm{N}$, $88^{\circ} 05^{\prime}$ to $89^{\circ} 55^{\prime} \mathrm{E}$ ) in India is the single largest mangrove in the world and has the greatest variability in the hydrological features both at a spatial and temporal scale. This mangrove was declared as a world heritage site by IUCN in 1987 for its rich faunal diversity. The topography and quality of the substratum, the salinity variations and the interactions among these factors result in a high habitat heterogeneity in the mangrove, ensuring a similarly diverse faunal community (Gopal and Chauhan 2006).

The habitat parameters (physical structure and edaphic and hydrological parameters) normally have a strong influence on the diversity and abundance of the associated organisms (MacArthur and MacArthur 1961, Lawton 1983, Ellner 2001). The complex system of prop-roots and pneumatophores in mangrove forests, in association with an often dense layer of leaf litter and detritus on the substratum, provides generous cover from predators for many invertebrate species (Acosta and Butler 1997, Primavera 1997, Laegdsgaard and Johnson 2001). In addition, variation in the density and structure of mangrove roots produces an array of shelters (Primavera 1997, Macia et al. 2001). Such variation normally affects the benthic fauna in a species-specific manner (Kon et al. 2009). Although some studies have examined effects of mangrove loss on benthic and epibenthic animals (Levings et al. 1994), few studies have examined how smallerscale modification of habitat complexity affects the biodiversity and abundance of mangrove-associated fauna (reviewed by Hatcher et al. 1989).
Among all benthic macro fauna in the intertidal zone, brachyurans are the most significant because of their great diversity, comprising about 1271 genera and 6793 species ( $\mathrm{Ng}$ et al. 2008). Brachyurans are also the most important macro-invertebrates as they make up $80 \%$ of the total faunal biomass (Golley et al. 1962) and attain high density (Macintosh 1984). Their role in the ecological functioning of the mangrove ecosystem (Lee 1998) through litter turnover (Robertson 1986) and bioturbation of soil (Smith et al. 1991) provides crucial food sources for coastal fisheries (Macintosh 1984). For all these reasons, Smith et al. (1991) recommended crabs as possible keystone species in the mangrove habitat. Except for the distribution and abundance of some of the dominant species of the Sundarban mangrove, no detailed information is available on the crab community. Therefore, the objectives of the present study were (i) to compare the community structure of brachyuran crabs at two mangrove sites with different habitat attributes because each habitat functions according to different physical, edaphic and hydrological properties, and (ii) to evaluate whether brachyuran crabs can be used as an indicator of habitat status in mangrove ecosystem.

\section{MATERIALS AND METHODS}

\section{Study sites}

The present study was conducted in the central part of Sundarban mangrove, namely at Jhorkhali Island (22 $\left.{ }^{\circ} 01^{\prime} 14.55^{\prime \prime} \mathrm{N}, 88^{\circ} 41^{\prime} 30.39^{\prime} \mathrm{E}\right)$ located between the two large rivers Matla and Bidya. Two study sites were selected; site- 1 along the mudflats of the Matla River and site-2 along the Boro Herobhanga creek (Fig. 1). Both sites experience a subtropical monsoonal climate with an annual rainfall of about 1600-1800 mm (Man-

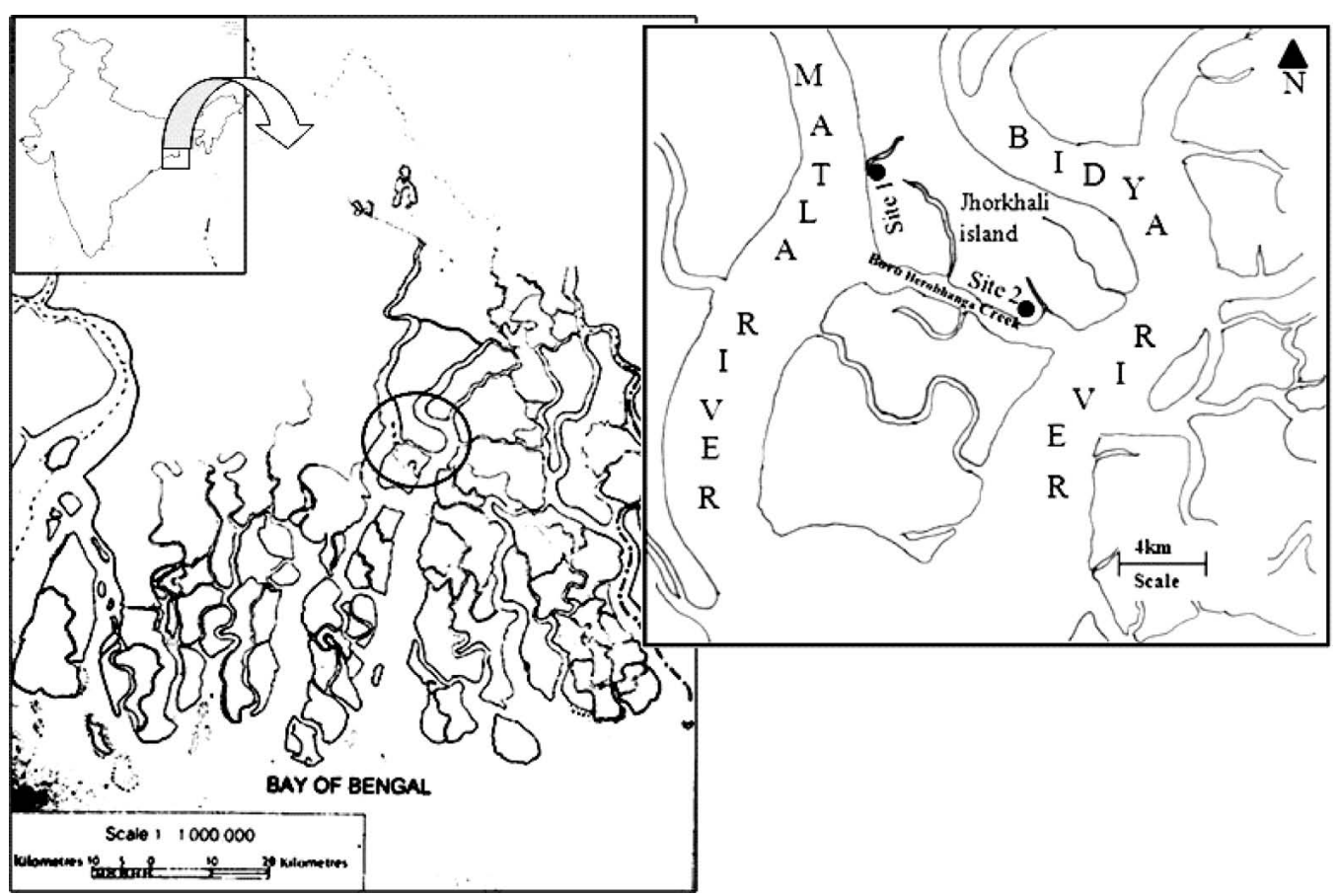

Fig. 1. - Map showing the Study sites as black dots in Jhorkhali Island, Sundarban. 
na et al. 2010). At site 1, the mangrove forest is truly natural without much anthropogenic intrusion, and with four dominant arboreal species (The dominant mangrove species are Avicennia marina, A. officinalis, Rhizophora apiculata and Excoecaria agallocha). Site 2 has a docking site for local ferry boat services and a mangrove replantation plot dating back to the 1990s, with a mixed vegetation composition ( $R$. apiculata, A. marina, A. officinalis, Bruguiera cylindrica, Haritiera fomes, Ceriops sp., Sonneratia alba, etc.). The replantation plot serves as a source for replantation programmes throughout Sundarban.

The sampling area of each site was $3 \mathrm{~km}^{2}$, within which three 1-km transects were laid (two along the two sides of the square and one along the middle). About sixty $2 \times 2 \mathrm{~m}^{2}$ quadrats were placed randomly along the transects for the collection of the data. In all the quadrats physical, hydrological and edaphic parameters were thoroughly analysed twice in a month for three seasons (pre-monsoon, monsoon and post-monsoon) in three consecutive years. For each of the variables, 10 replicates were made during each sampling. The number of the pneumatophores, senescent and decaying leaves, shrub and herb density were recorded in each quadrat. Percentage of canopy cover was determined using a locally built densiometer, while canopy height and shrub height were determined using measuring tape.

\section{Soil sampling design}

Soil samples were collected from each quadrat with the help of a soil corer and carried to the laboratory in plastic zipper pouches. In the laboratory, the initial weight of the soil samples was taken, the samples were oven dried $\left(60^{\circ} \mathrm{C}\right.$ for 48 hours) and the final weight of the samples was taken for the measurement of soil moisture. Soil $\mathrm{pH}$ and soil specific gravity were determined following the method of Brower et al. (1998). Soil organic carbon and organic matter were assessed using a modified Walkey and Black method (Nelson and Sommers, 1996).

\section{Water sampling design}

At both sites, subsurface water samples were collected twice every month simultaneously with crab sampling during high tide from a depth of 20-30 cm. Overall 13 hydrological variables were considered. Air temperature and water temperature were measured instantly with a mercury thermometer with $0.1^{\circ} \mathrm{C}$ graduations. Dissolved oxygen was measured at the site using a Lutton, DO-5509 Dissolved Oxygen Meter. Total Dissolved Solute and $\mathrm{pH}$ were also recorded in the field using portable testers (Eutech instrument, cyber scan). Water samples were collected in amber-coloured, labelled glass bottles for analysing the rest of the hydrological parameters. Samples were preserved at $4^{\circ} \mathrm{C}$ without freezing and taken to the laboratory within 3 $\mathrm{h}$ of collection for analysis as per standard methods (APHA 1998). In the laboratory, total alkalinity was measured following the acid titrimetric method, using $\mathrm{H}_{2} \mathrm{SO}_{4}$ as titrant and methyl orange as an indicator. Sa- linity was determined in practical salinity units (PSU) by the Knudsen argentometric method (Strickland and Parsons 1972). Total acidity was measured following the alkaline titrimetric method using $\mathrm{NaOH}$ as the titrant and phenolphthalein as the indicator. Total hardness was assayed by the EDTA titrimetric method. Inorganic phosphate, reactive silicate, nitrate-nitrogen and nitrite-nitrogen were measured using a UV spectrophotometer following standard sea water analysis methods (Grasshoff 1983, Grasshoff et al. 1983, Strickland and Parsons 1972).

\section{Crab sampling design}

During both low spring tide and low neap tide crabs were collected quantitatively by performing three individual time-based samplings in each of the $2 \times 2 \mathrm{~m}^{2}$ quadrats. The collection was based on the assumption that brachyuran crabs generally do not change significantly over the time scale (Ashton et al. 2003a). Precisely, three independent time-based samplings were employed in each quadrat. One sampling (considered as one replicate) represented one person collecting crabs for $15 \mathrm{~min}$. This method, first devised by Ashton et al. (2003a), was found to offer a good representative sample of the crab population residing at each site. Crabs were collected mostly by hand, sometimes with the help of a trowel. To reduce the bias in favour of slow-moving species, great efforts were given to catching the larger and fast-moving elusive species. After collection, the crabs were kept in $70 \%$ alcohol inside labelled plastic containers and carefully transported back to the laboratory. In the laboratory the crabs were identified and preserved in $4 \%$ formalin for future identification.

\section{Data analysis}

As the study focuses on the temporal factors (seasons and years) at two different study sites, one-way analysis of variance (ANOVA) at 5\% level of significance was used to test for significance for the environmental parameters followed by Duncan's post hoc tests to check for differences in the environmental factors between every pair of seasons and year. These parts of the statistical analyses were carried out by means of SPSS (Statistical Package for Social Sciences, version 17.0 Norušis 2000).

A total of 2055 individuals were collected during the period of three years. Total crab abundance was calculated at two different sites for three seasons in each year: pre-monsoon, monsoon and post-monsoon. From the sampled data, Pielou evenness (J) (Pielou 1969) and species dominance (D) index (Berger and Parker 1970) were calculated for two sites in three seasons each year. Univariate measures were transformed using square-root to normality, if necessary. The similarity in seasonal species composition at each site was studied by calculating the Bray-Curtis coefficient based on the square-root-transformed species abundance data. The result was displayed by non-metric multidimensional scaling (nMDS) plot (Clarke 1993). For differences 
Table 1. - Hydrological parameters of study sites in the Indian Sundarbans. SAL, salinity (PSU); ACI, total acidity (mg L ${ }^{-1}$ ); ALK, total alkalinity $\left(\mathrm{mg} \mathrm{L}^{-1}\right)$; $\mathrm{TH}$, total hardness $\left(\mathrm{mg} \mathrm{L}^{-1}\right)$; WT, water temperature $\left({ }^{\circ} \mathrm{C}\right)$; AT, air temperature $\left({ }^{\circ} \mathrm{C}\right)$; $\mathrm{pH}$; TDS, total dissolved solids $(\mathrm{ppm})$; inorganic phosphate $\left(\mu \mathrm{M} \mathrm{L}^{-1}\right)$; NO3-N, nitrate-nitrogen $\left(\mu \mathrm{M} \mathrm{L}^{-1}\right)$; NO2-N, nitrite-nitrogen $\left(\mu \mathrm{M} \mathrm{L}^{-1}\right)$. Different letters indicate significant differences at $0.05 \%$ level of significance.

\begin{tabular}{|c|c|c|c|c|c|c|c|c|c|c|c|c|c|}
\hline & & & SAL & ACI & ALK & $\mathrm{TH}$ & WT & AT & $\mathrm{pH}$ & TDS & Ip & NO3-N & $\mathrm{NO} 2-\mathrm{N}$ \\
\hline \multirow{6}{*}{ Year 1} & \multirow[b]{2}{*}{ PMo } & St1 & $\begin{array}{l}27.42^{\mathrm{a}} \\
\pm 0.32\end{array}$ & $\begin{array}{c}18.15^{\mathrm{a} 1} \\
\pm 0.10\end{array}$ & $\begin{array}{c}139.18^{\mathrm{a} 2} \\
\pm 0.11\end{array}$ & $\begin{array}{c}120.24^{\mathrm{a} 3} \\
\pm 0.04\end{array}$ & $\begin{array}{c}30.38^{\mathrm{a} 4} \\
\pm 0.06\end{array}$ & $\begin{array}{c}33.33^{\mathrm{a} 5} \\
\pm 0.08\end{array}$ & $\begin{array}{l}8.20^{\mathrm{a} 6} \\
\pm 0.04\end{array}$ & $\begin{array}{c}1448.48^{\mathrm{a} 7} \\
\pm 0.27\end{array}$ & $\begin{array}{l}0.26^{\mathrm{a} 8} \\
\pm 0.00\end{array}$ & $\begin{array}{l}0.93^{\mathrm{a} 9} \\
\pm 0.03\end{array}$ & $\begin{array}{c}0.48^{\mathrm{a} 10} \\
\pm 0.00\end{array}$ \\
\hline & & St2 & $\begin{array}{c}25.91^{\mathrm{a}} \\
\pm 0.23\end{array}$ & $\begin{array}{c}31.87^{\mathrm{al}} \\
\pm 0.35\end{array}$ & $\begin{array}{c}165.00^{\mathrm{a} 2} \\
\pm 1.86\end{array}$ & $\begin{array}{c}181.14^{\mathrm{a} 3}{ }^{\prime} \\
\pm 3.08\end{array}$ & $\begin{array}{c}32.58^{\mathrm{a} 4} \\
\pm 0.09\end{array}$ & $\begin{array}{c}36.35^{\mathrm{a} 5} \\
\pm 0.06\end{array}$ & $\begin{array}{l}7.63^{\mathrm{a} 6} \\
\pm 0.06\end{array}$ & $\begin{array}{c}1437.22^{\mathrm{a} 7^{\prime}} \\
\pm 0.90\end{array}$ & $\begin{array}{l}0.07^{\mathrm{a} 8} \\
\pm 0.00\end{array}$ & $\begin{array}{l}0.87^{\mathrm{a} 9} \\
\pm 0.01\end{array}$ & $\begin{array}{c}0.18^{\mathrm{a} 10} \\
\pm 0.01\end{array}$ \\
\hline & \multirow[b]{2}{*}{ Mo } & St1 & $\begin{array}{l}15.49^{b} \\
\pm 0.88\end{array}$ & $\begin{array}{c}44.88^{\mathrm{b} 1} \\
\pm 0.23\end{array}$ & $\begin{array}{c}117.99^{\mathrm{b} 2} \\
\pm 0.34\end{array}$ & $\begin{array}{c}56.43^{\mathrm{b} 3} \\
\pm 0.14\end{array}$ & $\begin{array}{c}27.52^{\mathrm{b} 4} \\
\pm 0.19\end{array}$ & $\begin{array}{c}31.52^{\mathrm{b} 5} \\
\pm 0.12\end{array}$ & $\begin{array}{l}7.95^{\mathrm{b} 6} \\
\pm 0.03\end{array}$ & $\begin{array}{c}1675.24^{\mathrm{b} 7} \\
\pm 3.00\end{array}$ & $\begin{array}{l}2.30^{\mathrm{a} 8} \\
\pm 0.07\end{array}$ & $\begin{array}{l}2.55^{\mathrm{a} 9} \\
\pm 0.07\end{array}$ & $\begin{array}{c}0.57^{\mathrm{a} 10} \\
\pm 0.01\end{array}$ \\
\hline & & St2 & $\begin{array}{c}21.27^{\mathrm{b}} \\
\pm 0.61\end{array}$ & $\begin{array}{c}63.06^{\mathrm{b} 1} \\
\pm 0.53\end{array}$ & $\begin{array}{c}160.52^{\mathrm{b} 2} \\
\pm 0.25\end{array}$ & $\begin{array}{c}76.16^{\mathrm{b} 3} \\
\pm 0.85\end{array}$ & $\begin{array}{c}31.60^{\mathrm{b} 4} \\
\pm 0.09\end{array}$ & $\begin{array}{c}35.28^{b 5} \\
\pm 0.05\end{array}$ & $\begin{array}{l}7.45^{\mathrm{b} 6} \\
\pm 0.03\end{array}$ & $\begin{array}{c}1653.94^{\mathrm{b} 7} \\
\pm 1.57\end{array}$ & $\begin{array}{l}1.05^{\mathrm{b} 8} \\
\pm 0.01\end{array}$ & $\begin{array}{l}1.62^{\mathrm{b} 9} \\
\pm 0.12\end{array}$ & $\begin{array}{c}0.18^{\mathrm{b} 10^{\prime}} \\
\pm 0.01\end{array}$ \\
\hline & \multirow[b]{2}{*}{ PoMo } & St1 & $\begin{array}{l}23.81^{\mathrm{c}} \\
\pm 1.01^{1}\end{array}$ & $\begin{array}{c}36.85^{\mathrm{c} 1} \\
\pm 0.23\end{array}$ & $\begin{array}{c}125.68^{\mathrm{c} 2} \\
\pm 0.47\end{array}$ & $\begin{array}{c}62.31^{\mathrm{c} 3} \\
\pm 0.08\end{array}$ & $\begin{array}{c}22.65^{\mathrm{c} 4} \\
\pm 0.09\end{array}$ & $\begin{array}{c}26.45^{\mathrm{c} 5} \\
\pm 0.13\end{array}$ & $\begin{array}{l}7.17^{\mathrm{c} 6} \\
\pm 0.01\end{array}$ & $\begin{array}{c}2334.63^{\mathrm{c} 7} \\
\pm 1.59\end{array}$ & $\begin{array}{l}1.35^{\mathrm{a} 8} \\
\pm 0.01\end{array}$ & $\begin{array}{l}3.37^{\mathrm{a} 9} \\
\pm 0.07\end{array}$ & $\begin{array}{c}0.86^{\mathrm{a} 10} \\
\pm 0.01\end{array}$ \\
\hline & & St2 & $\begin{array}{c}27.99^{c^{\prime}} \\
\pm 0.21\end{array}$ & $\begin{array}{c}57.97^{\mathrm{c} 1} \\
\pm 0.33\end{array}$ & $\begin{array}{c}195.77^{\mathrm{c} 2} \\
\pm 1.26\end{array}$ & $\begin{array}{c}133.50^{\mathrm{c} 3} \\
\pm 16.3\end{array}$ & $\begin{array}{c}26.45^{\mathrm{c} 4} \\
\pm 0.06\end{array}$ & $\begin{array}{c}30.20^{\mathrm{c} 5} \\
\pm 0.07\end{array}$ & $\begin{array}{l}6.78^{\mathrm{c} 6} \\
\pm 0.02\end{array}$ & $\begin{array}{c}2434.52^{\mathrm{c} 7} \\
\pm 10.24\end{array}$ & $\begin{array}{l}0.94^{\mathrm{c} 8} \\
\pm 0.01\end{array}$ & $\begin{array}{l}1.37^{\mathrm{c} 9} \\
\pm 0.03\end{array}$ & $\begin{array}{c}0.28^{\mathrm{c} 10^{\prime}} \\
\pm 0.01\end{array}$ \\
\hline \multirow{6}{*}{ Year 2} & \multirow[b]{2}{*}{ PMo } & St1 & $\begin{array}{l}31.35^{\mathrm{a}} \\
\pm 0.42\end{array}$ & $\begin{array}{c}27.99 \mathrm{~d} 1 \\
\pm 0.26\end{array}$ & $\begin{array}{c}112.58^{\mathrm{d} 2} \\
\pm 0.24\end{array}$ & $\begin{array}{c}43.50^{\mathrm{d} 3} \\
\pm 1.19\end{array}$ & $\begin{array}{c}33.08^{\mathrm{d} 4} \\
\pm 0.81\end{array}$ & $\begin{array}{c}36.48^{\mathrm{a} 5} \\
\pm 0.03\end{array}$ & $\begin{array}{l}7.30 \mathrm{~d} 6 \\
\pm 0.07\end{array}$ & $\begin{array}{c}1161.62^{\mathrm{d} 7} \\
\pm 3.25\end{array}$ & $\begin{array}{l}1.45^{\mathrm{a} 8} \\
\pm 0.01\end{array}$ & $\begin{array}{l}3.20^{\mathrm{d} 9} \\
\pm 0.00\end{array}$ & $\begin{array}{l}0.84^{\mathrm{d} 10} \\
\pm 0.01\end{array}$ \\
\hline & & $\mathrm{St} 2$ & $\begin{array}{c}26.93^{\mathrm{a}} \\
\pm 0.33\end{array}$ & $\begin{array}{c}64.01^{\mathrm{d} 1} \\
\pm 0.60\end{array}$ & $\begin{array}{c}156.81^{\mathrm{d} 2} \\
\pm 1.15\end{array}$ & $\begin{array}{c}91.27 \mathrm{~d} 3 \\
\pm 0.98\end{array}$ & $\begin{array}{c}36.63^{\mathrm{a} 4} \\
\pm 0.10\end{array}$ & $\begin{array}{c}38.45^{\mathrm{a} 5} \\
\pm 0.06\end{array}$ & $\begin{array}{l}6.78^{\mathrm{d} 6} \\
\pm 0.09\end{array}$ & $\begin{array}{c}1133.23^{\mathrm{d} 7} \\
\pm 0.71\end{array}$ & $\begin{array}{l}0.48^{\mathrm{a} 8} \\
\pm 0.00\end{array}$ & $\begin{array}{c}2.17^{\mathrm{d} 9} \\
\pm 0.01\end{array}$ & $\begin{array}{c}0.45^{\mathrm{b} 10} \\
\pm 0.01\end{array}$ \\
\hline & \multirow[b]{2}{*}{ Mo } & St1 & $\begin{array}{l}13.99 \mathrm{e} \\
\pm 0.50\end{array}$ & $\begin{array}{c}57.92^{\mathrm{b} 1} \\
\pm 0.21\end{array}$ & $\begin{array}{c}100.71^{\mathrm{b} 2} \\
\pm 0.43\end{array}$ & $\begin{array}{c}38.48^{\mathrm{b} 3} \\
\pm 0.41\end{array}$ & $\begin{array}{c}28.35^{\mathrm{e} 4} \\
\pm 0.03\end{array}$ & $\begin{array}{c}34.23^{\mathrm{e} 5} \\
\pm 0.10\end{array}$ & $\begin{array}{l}7.20^{\mathrm{b} 6} \\
\pm 0.04\end{array}$ & $\begin{array}{c}1345.91^{\mathrm{e} 7} \\
\pm 1.31\end{array}$ & $\begin{array}{l}3.75^{\mathrm{a} 8} \\
\pm 0.03\end{array}$ & $\begin{array}{c}3.08 \mathrm{e} 9 \mathrm{a} 9 \\
\pm 0.03\end{array}$ & $\begin{array}{c}1.34^{\mathrm{d} 10 \mathrm{a} 10} \\
\pm 0.01\end{array}$ \\
\hline & & $\mathrm{St} 2$ & $\begin{array}{c}21.99^{e} \\
\pm 0.26\end{array}$ & $\begin{array}{c}95.96^{\mathrm{b} 1} \text { 'b } 1 \\
\pm 0.38\end{array}$ & $\begin{array}{c}118.42^{\mathrm{e} 2} \\
\pm 0.48\end{array}$ & $\begin{array}{c}64.50^{\mathrm{b} 3} \\
\pm 0.47\end{array}$ & $\begin{array}{c}32.23^{\mathrm{e} 4^{\prime}} \\
\pm 0.06\end{array}$ & $\begin{array}{c}36.28^{\mathrm{e} 5} \\
\pm 0.09\end{array}$ & $\begin{array}{l}6.40^{\mathrm{e}} 6^{\prime} \\
\pm 0.04\end{array}$ & $\begin{array}{c}1338.13^{\mathrm{e} 7} \\
\pm 0.24\end{array}$ & $\begin{array}{c}0.88^{\mathrm{a} 8}{ }^{\prime} \mathrm{b} 8 \\
\pm 0.00\end{array}$ & $\begin{array}{c}1.64^{\mathrm{e} 9} \mathrm{\prime} 9^{\prime} \\
\pm 0.01\end{array}$ & $\begin{array}{c}0.66^{\mathrm{b} 10} \\
\pm 0.02\end{array}$ \\
\hline & \multirow[b]{2}{*}{ PoMo } & St1 & $\begin{array}{l}15.03^{\mathrm{f}} \\
\pm 0.47\end{array}$ & $\begin{array}{c}56.96^{\mathrm{cl}} \\
\pm 0.37\end{array}$ & $\begin{array}{c}129.75^{\mathrm{f} 2} \\
\pm 1.60\end{array}$ & $\begin{array}{c}27.12^{\mathrm{f} 3} \\
\pm 0.47\end{array}$ & $\begin{array}{c}26.58^{\mathrm{f} 4} \\
\pm 0.08\end{array}$ & $\begin{array}{c}29.23^{\mathrm{f} 5} \\
\pm 0.06\end{array}$ & $\begin{array}{l}7.28^{\mathrm{f} 6} \\
\pm 0.05\end{array}$ & $\begin{array}{c}2489.97^{\mathrm{f} 7} \\
\pm 4.50\end{array}$ & $\begin{array}{l}3.45^{\mathrm{a} 8} \\
\pm 0.04\end{array}$ & $\begin{array}{c}3.53^{\mathrm{f} 9 \mathrm{a} 9} \\
\pm 0.03\end{array}$ & $\begin{array}{c}0.97^{\mathrm{d} 10} \\
\pm 0.01\end{array}$ \\
\hline & & St2 & $\begin{array}{c}28.28^{\mathrm{f}} \\
\pm 0.22\end{array}$ & $\begin{array}{c}86.21^{\mathrm{c} 1} \\
\pm 0.29\end{array}$ & $\begin{array}{c}135.59^{\mathrm{f} 2} \\
\pm 1.12\end{array}$ & $\begin{array}{c}88.52^{\mathrm{f} 3} \\
\pm 0.35\end{array}$ & $\begin{array}{c}29.58^{\mathrm{f} 4} \\
\pm 0.14\end{array}$ & $\begin{array}{c}34.85^{\mathrm{f5}} \\
\pm 0.24\end{array}$ & $\begin{array}{l}6.75^{\mathrm{f} 6} \\
\pm 0.03\end{array}$ & $\begin{array}{c}2440.80^{\mathrm{f} 7} \\
\pm 0.25\end{array}$ & $\begin{array}{c}1.19^{\mathrm{a} 8}{ }^{\prime} \mathrm{c} 8^{\prime} \\
\pm 0.00\end{array}$ & $\begin{array}{c}2.45^{\mathrm{f} 9^{\prime} \mathrm{c} 9} \\
\pm 0.02\end{array}$ & $\begin{array}{c}0.53^{\mathrm{b} 10} \mathrm{c} 10^{\prime} \\
\pm 0.00\end{array}$ \\
\hline \multirow{6}{*}{ Year 3} & \multirow{2}{*}{ PMo } & St1 & $\begin{array}{l}27.80^{\mathrm{a}} \\
\pm 0.47\end{array}$ & $\begin{array}{c}63.16^{\mathrm{g} 1} \\
\pm 0.43\end{array}$ & $\begin{array}{c}88.27 \mathrm{~g} 2 \\
\pm 0.06\end{array}$ & $\begin{array}{c}22.71^{\mathrm{g} 3} \\
\pm 0.28\end{array}$ & $\begin{array}{c}29.50^{\mathrm{a} 4} \\
\pm 0.04\end{array}$ & $\begin{array}{c}34.38^{\mathrm{a} 5} \\
\pm 0.10\end{array}$ & $\begin{array}{l}7.21^{\mathrm{g} 6} \\
\pm 0.00\end{array}$ & $\begin{array}{c}1557.02^{\mathrm{g} 7} \\
\pm 0.47\end{array}$ & $\begin{array}{l}2.84^{\mathrm{a} 8} \\
\pm 0.02\end{array}$ & $\begin{array}{l}3.91^{\mathrm{g} 9} \\
\pm 0.02\end{array}$ & $\begin{array}{l}1.70^{\mathrm{g} 10} \\
\pm 0.15\end{array}$ \\
\hline & & $\mathrm{St} 2$ & $\begin{array}{c}32.00^{\mathrm{a}} \\
\pm 0.27\end{array}$ & $\begin{array}{c}77.99 \mathrm{~g} 1 \\
\pm 0.18\end{array}$ & $\begin{array}{c}144.62^{\mathrm{g} 2} \\
\pm 1.14\end{array}$ & $\begin{array}{c}107.97^{\mathrm{g} 3} \\
\pm 1.44\end{array}$ & $\begin{array}{c}33.28^{\mathrm{a} 4} \\
\pm 0.07\end{array}$ & $\begin{array}{c}32.28^{\mathrm{a} 5} \\
\pm 0.09\end{array}$ & $\begin{array}{l}7.04^{\mathrm{g} 6} \\
\pm 0.07\end{array}$ & $\begin{array}{c}1180.24 \mathrm{~g} 7 \\
\pm 7.35\end{array}$ & $\begin{array}{l}0.85^{\mathrm{a} 8} \\
\pm 0.00\end{array}$ & $\begin{array}{l}1.69 \mathrm{~g} 9 \\
\pm 0.09\end{array}$ & $\begin{array}{c}0.63^{\mathrm{g} 10} \\
\pm 0.00\end{array}$ \\
\hline & \multirow[b]{2}{*}{ Mo } & St1 & $\begin{array}{l}9.96^{\mathrm{h}} \\
\pm 0.43\end{array}$ & $\begin{array}{c}86.66^{\mathrm{b} 1} \\
\pm 0.24\end{array}$ & $\begin{array}{c}90.26^{\mathrm{b} 2} \\
\pm 0.04\end{array}$ & $\begin{array}{c}32.29^{\mathrm{b} 3} \\
\pm 0.13\end{array}$ & $\begin{array}{c}27.65^{\mathrm{h} 4} \\
\pm 0.12\end{array}$ & $\begin{array}{c}32.33^{\mathrm{h} 5} \\
\pm 0.11\end{array}$ & $\begin{array}{l}7.53^{\mathrm{b} 6} \\
\pm 0.11\end{array}$ & $\begin{array}{c}1473.10^{\mathrm{h} 7} \\
\pm 5.32\end{array}$ & $\begin{array}{c}4.07^{\mathrm{h} 8 \mathrm{a} 8} \\
\pm 0.01\end{array}$ & $\begin{array}{c}8.46^{\mathrm{h} 9 \mathrm{a} 9} \\
\pm 0.07\end{array}$ & $\begin{array}{l}2.78^{\text {h } 10 \mathrm{a} 10} \\
\pm 0.07\end{array}$ \\
\hline & & $\mathrm{St} 2$ & $\begin{array}{c}12.30^{\mathrm{h}} \\
\pm 0.11\end{array}$ & $\begin{array}{c}94.43^{\mathrm{g} 1} \\
\pm 0.32\end{array}$ & $\begin{array}{c}122.06^{\mathrm{b} 2} \\
\pm 1.48\end{array}$ & $\begin{array}{c}110.83^{\mathrm{b} 3} \\
\pm 3.49\end{array}$ & $\begin{array}{c}31.50^{\mathrm{h} 4} \\
\pm 0.10\end{array}$ & $\begin{array}{c}34.83^{\mathrm{h} 5} \\
\pm 0.36\end{array}$ & $\begin{array}{l}6.73^{\mathrm{h} 6} \\
\pm 0.09\end{array}$ & $\begin{array}{c}1348.62^{\mathrm{h} 7} \\
\pm 3.58\end{array}$ & $\begin{array}{c}0.98^{g} 8^{\prime} \mathrm{b} 8^{\prime} \\
\pm 0.00\end{array}$ & $\begin{array}{c}3.25^{\mathrm{h} 9{ }^{\prime} \mathrm{b} 9} \\
\pm 0.00\end{array}$ & $\begin{array}{c}1.45^{\mathrm{b} 10} \\
\pm 0.18\end{array}$ \\
\hline & \multirow[b]{2}{*}{ PoMo } & St1 & $\begin{array}{l}15.59^{i} \\
\pm 0.40\end{array}$ & $\begin{array}{c}81.16^{\mathrm{c} 1} \\
\pm 0.41\end{array}$ & $\begin{array}{c}101.57^{\mathrm{i} 2} \\
\pm 0.26\end{array}$ & $\begin{array}{c}18.26^{\mathrm{i} 3} \\
\pm 0.02\end{array}$ & $\begin{array}{c}23.33^{\mathrm{i} 4} \\
\pm 0.11\end{array}$ & $\begin{array}{c}28.05^{\mathrm{i} 5} \\
\pm 0.29\end{array}$ & $\begin{array}{l}7.13^{i 6} \\
\pm 0.03\end{array}$ & $\begin{array}{c}1520.93^{\mathrm{i} 7} \\
\pm 2.89\end{array}$ & $\begin{array}{l}2.84^{\mathrm{i} 8} \\
\pm 0.02\end{array}$ & $\begin{array}{l}8.50^{\mathrm{i} 9 \mathrm{a} 9} \\
\pm 0.05\end{array}$ & $\begin{array}{l}3.45^{\mathrm{i} 10} \\
\pm 0.09\end{array}$ \\
\hline & & St2 & $\begin{array}{l}21.36^{\mathrm{i}} \\
\pm 0.04\end{array}$ & $\begin{array}{c}84.10^{\mathrm{g} 1} \\
\pm 0.30\end{array}$ & $\begin{array}{c}118.49^{\mathrm{i} 2^{\prime}} \\
\pm 0.09\end{array}$ & $\begin{array}{c}114.80^{\mathrm{i} 3} \\
\pm 1.29\end{array}$ & $\begin{array}{c}29.08^{\mathrm{i} 4} \\
\pm 0.30\end{array}$ & $\begin{array}{c}33.58^{\mathrm{i} 5} \\
\pm 0.09\end{array}$ & $\begin{array}{l}6.45^{\mathrm{i} 6^{\prime}} \\
\pm 0.13\end{array}$ & $\begin{array}{c}1144.96^{\mathrm{i} 7} \\
\pm 1.60\end{array}$ & $\begin{array}{c}1.27^{\mathrm{g} 8} \mathrm{c} \mathrm{c}^{\prime} \\
\pm 0.01\end{array}$ & $\begin{array}{c}5.35^{\mathrm{i} 9^{\prime} \mathrm{c} 9} \\
\pm 0.11\end{array}$ & $\begin{array}{l}1.39^{\mathrm{c} 10} \\
\pm 0.05\end{array}$ \\
\hline
\end{tabular}

between the study sites, formal significance tests for similarity were performed using the one-way ANOSIM permutation test on the crab similarity matrix. The crab species which are contributing to the dissimilarities between the two study sites were investigated using the similarity percentage (SIMPER) (Clarke and Warwick 1994). All mentioned statistical treatments were performed using PRIMER-E Software (v. 6).

In order to assess the interaction between different hydrological, edaphic and physical parameters and the crab species assemblage, unimodal distribution of species was used to explain the abundance of species with environmental variables (water temperature, $\mathrm{pH}$, salinity, total acidity, total alkalinity, total hardness, inorganic phosphate, nitrate-nitrogen and nitritenitrogen, soil organic carbon, soil organic matter, soil $\mathrm{pH}$, soil density, soil specific gravity, \% of canopy cover, canopy height, pneumatophore density, $\%$ of leaf litter, herb density, etc.). Canonical correspondence analysis (CCA) was employed with biplot type of scaling on inter-species distance and down weighting of rare species. The species abundance data were square-root transformed. A test of significance of the first canonical axis and all canonical axes was made using the Monte Carlo test with 499 permutations under the reduced model (Ter Braak 1986, 1988, Jongman et al. 1995). The relationships of crab species abundance with environmental variables were presented in ordination diagrams (biplots) for the first two axes using CANOCO 4.5 software package.

\section{RESULTS}

Seasonal and annual trends in hydrological and edaphic parameters for the two sites are given in Table 1 and Table 2, respectively. Most of the parameters, and specially inorganic phosphate, nitrite-nitrogen, nitrate-nitrogen, salinity of water and organic carbon along with organic matter and moisture content of soil, differed significantly $(p<0.05)$ between the two sites. At site 1, all the hydrological parameters and most of the edaphic factors varied significantly $(p<0.05)$ between three seasons of a year and also between years of a particular season. At site 2, hydrological and edaphic parameters of the habitat differed $(p<0.05)$ mostly in all the seasons and in all three years of a particular season 
Table 2. - Edaphic parameters of study sites in the Indian Sundarbans; soil organic carbon (\%); soil organic matter (\%); soil moisture (\%); soil $\mathrm{pH}$; soil density and soil specific gravity. Different letters indicate significant differences at $0.05 \%$ level of significance.

\begin{tabular}{|c|c|c|c|c|c|c|c|c|}
\hline & & & $\begin{array}{c}\text { Soil organic } \\
\text { carbon }\end{array}$ & $\begin{array}{c}\text { Soil organic } \\
\text { matter }\end{array}$ & Soil moisture & Soil pH & Soil density & $\begin{array}{c}\text { Soil specific } \\
\text { gravity }\end{array}$ \\
\hline \multirow{6}{*}{ Year 1} & \multirow[b]{2}{*}{ PMo } & St1 & $1.00^{\mathrm{a} 1} \pm 0.00$ & $1.73^{\mathrm{a} 2} \pm 0.00$ & $42.47^{\mathrm{a} 3} \pm 0.17$ & $7.58^{\mathrm{a} 4} \pm 0.11$ & $2.73^{\mathrm{a} 5} \pm 0.07$ & $1.39^{\mathrm{a}} \pm 0.04$ \\
\hline & & St2 & $0.44^{\mathrm{a} 1} \pm 0.00$ & $0.76^{\mathrm{a} 2} \pm 0.00$ & $46.91^{\mathrm{a} 3^{\prime}} \pm 0.45$ & $7.75^{\mathrm{a} 4} \pm 0.01$ & $2.58^{\mathrm{a} 5} \pm 0.05$ & $1.48^{\mathrm{a} 6} \pm 0.08$ \\
\hline & \multirow{2}{*}{ Mo } & St1 & $1.03^{\mathrm{b} 1} \pm 0.00$ & $1.77^{\mathrm{a} 2} \pm 0.00$ & $53.10^{\mathrm{b} 3} \pm 3.80$ & $7.57^{\mathrm{b} 4} \pm 0.10$ & $2.90^{\mathrm{b} 5} \pm 0.02$ & $1.46^{\mathrm{b} 6} \pm 0.02$ \\
\hline & & St2 & $0.76^{\mathrm{al}^{\prime} \pm 0.01}$ & $1.30^{\mathrm{a} 2} \pm 0.02$ & $59.54^{\mathrm{b} 3} \pm 0.42$ & $7.47^{\mathrm{b} 4 \mathrm{a} 4} \pm 0.02$ & $1.69^{\mathrm{b} 5} \pm 0.08$ & $1.23^{\mathrm{b} 6} \pm 0.00$ \\
\hline & \multirow{2}{*}{ PoMo } & St1 & $1.04^{\mathrm{c} 1} \pm 0.00$ & $1.79^{\mathrm{a} 2} \pm 0.00$ & $43.73^{\mathrm{c} 3} \pm 0.58$ & $7.54^{\mathrm{c} 4} \pm 0.03$ & $2.98^{\mathrm{c} 5} \pm 0.05$ & $1.36^{\mathrm{c} 6} \pm 0.02$ \\
\hline & & St2 & $0.94^{\mathrm{a} 1^{\prime}} \pm 0.01$ & $1.62^{\mathrm{a} 2^{\prime}} \pm 0.01$ & $34.87^{\mathrm{c} 3} \pm 0.29$ & $6.71^{\mathrm{c} 4^{\prime} \mathrm{a} 4} \pm 0.08$ & $1.73^{\mathrm{c} 5} \pm 0.01$ & $1.09^{c 6^{\prime}} \pm 0.03$ \\
\hline \multirow{6}{*}{ Year 2} & \multirow[b]{2}{*}{ PMo } & St1 & $0.95^{\mathrm{d} 1} \pm 0.02$ & $1.63^{\mathrm{d} 2} \pm 0.02$ & $40.44^{\mathrm{a} 3} \pm 3.80$ & $7.67^{\mathrm{d} 4} \pm 0.04$ & $1.80^{\mathrm{d} 5} \pm 0.13$ & $1.24^{\mathrm{d} 6} \pm 0.00$ \\
\hline & & St2 & $0.54^{\mathrm{d} 1} \pm 0.10$ & $0.92^{\mathrm{d} 2} \pm 0.17$ & $48.41^{\mathrm{d} 3} \pm 0.40$ & $7.88^{\mathrm{d} 4} \pm 0.04$ & $2.67^{\mathrm{a} 5^{5}} \pm 0.15$ & $1.24^{\mathrm{a} 6} \pm 0.01$ \\
\hline & \multirow[b]{2}{*}{ Mo } & St1 & $1.31^{\mathrm{e} 1} \pm 0.08$ & $2.26^{\mathrm{e} 2} \pm 0.15$ & $53.65^{\mathrm{e} 3 \mathrm{~b} 3} \pm 0.67$ & $7.67^{\mathrm{b} 4} \pm 0.01$ & $2.35^{\mathrm{b} 5} \pm 0.01$ & $1.05^{\mathrm{e} 6 \mathrm{~b} 6} \pm 0.00$ \\
\hline & & St2 & $0.88^{\mathrm{al}} \pm 0.01$ & $1.52^{\mathrm{a} 2} \mathrm{e}^{2} \pm 0.01$ & $54.89^{\mathrm{e} 3 \mathrm{~b} 3} \pm 0.24$ & $7.63^{\mathrm{b} 4} \pm 0.02$ & $1.49^{\mathrm{b} 5} \pm 0.09$ & $1.17^{\mathrm{e} 6^{6} \mathrm{~b} 6} \pm 0.01$ \\
\hline & \multirow{2}{*}{ PoMo } & St1 & $1.36^{\mathrm{al}} \pm 0.01$ & $2.35^{\mathrm{f} 2} \pm 0.02$ & $48.30^{\mathrm{f} 3} \pm 0.16$ & $7.73^{\mathrm{f} 4} \pm 0.05$ & $3.03^{\mathrm{f} 5} \pm 0.08$ & $1.58^{\mathrm{c} 6} \pm 0.02$ \\
\hline & & St2 & $1.19^{\mathrm{al}} \pm 0.00$ & $2.04^{\mathrm{f} 2 \mathrm{a} 2} \pm 0.00$ & $32.56^{\mathrm{f3}} \pm 0.09$ & $6.61^{\mathrm{f} 4 \mathrm{c} 4} \pm 0.05$ & $1.68^{\mathrm{f5}} \pm 0.01$ & $1.07^{\mathrm{f6}} \pm 0.02$ \\
\hline \multirow{6}{*}{ Year 3} & \multirow[b]{2}{*}{ PMo } & St1 & $0.95^{\mathrm{g} 1} \pm 0.02$ & $1.64^{\mathrm{g} 2} \pm 0.04$ & $45.77^{\mathrm{a} 3} \pm 7.78$ & $7.10^{\mathrm{g} 4} \pm 0.20$ & $1.78^{\mathrm{g} 5} \pm 0.08$ & $1.10^{\mathrm{g} 6} \pm 0.03$ \\
\hline & & St2 & $0.76^{\mathrm{g} 1} \pm 0.01$ & $1.32^{\mathrm{g} 2} \pm 0.02$ & $48.44^{\mathrm{g} 3} \pm 0.64$ & $7.77^{\mathrm{g} 4} \pm 0.05$ & $2.37^{\mathrm{a} 5} \pm 0.08$ & $1.24^{\mathrm{c} 6 \mathrm{a} 6^{\prime}} \pm 0.00$ \\
\hline & \multirow[b]{2}{*}{ Mo } & St1 & $1.24^{\mathrm{h} 1} \pm 0.01$ & $2.14^{\mathrm{h} 2} \pm 0.01$ & $57.12^{\mathrm{h} 3 \mathrm{~b} 3} \pm 1.05$ & $7.67^{\mathrm{h} 4 \mathrm{~b} 4} \pm 0.01$ & $2.38^{\mathrm{b} 5} \pm 0.02$ & $1.05^{\mathrm{h} 6 \mathrm{~b} 6} \pm 0.00$ \\
\hline & & St2 & $0.98^{\mathrm{al}} \pm 0.00$ & $1.69^{\mathrm{a} 2} \pm 0.01$ & $36.15^{\mathrm{h} 3 \mathrm{~b} 3} \pm 0.41$ & $6.77^{\mathrm{h} 4^{\prime} \mathrm{b} 4} \pm 0.07$ & $1.54^{\mathrm{h} 5} \pm 0.01$ & $1.02^{\mathrm{h} 6^{\prime} \mathrm{c} 6} \pm 0.00$ \\
\hline & \multirow[b]{2}{*}{ PoMo } & St1 & $1.33^{\mathrm{i} 1} \pm 0.07$ & $2.29^{\mathrm{i} 2} \pm 0.12$ & $43.19^{\mathrm{i} 3} \pm 0.20$ & $7.90^{\mathrm{i} 4} \pm 0.03$ & $2.65^{\mathrm{i} 5} \pm 0.12$ & $1.60^{\mathrm{i} 6 \mathrm{c} 6} \pm 0.03$ \\
\hline & & St2 & $1.27^{\mathrm{al}} \pm 0.01$ & $2.19^{\mathrm{a} 2} \pm 0.02$ & $38.39^{13} \pm 0.08$ & $7.22^{\mathrm{i} 4 \mathrm{c} 4} \pm 0.09$ & $1.97^{\mathrm{i} 5^{5}} \pm 0.01$ & $1.34^{\mathrm{i} 6^{\prime} \mathrm{c}{ }^{6}} \pm 0.01$ \\
\hline
\end{tabular}

with the exception of soil density in the pre-monsoon and monsoon seasons. Most of the environmental parameters were correlated with each other according to Spearman's correlation coefficient. Soil moisture was negatively correlated with number of senescent or decaying leaves $(r=-0.61, p<0.01)$, whereas the number of fallen leaves was positively correlated with pneumatophore density, canopy height and shrub height $(\mathrm{r}=$ 0.764, $\mathrm{p}<0.01, \mathrm{r}=0.639, \mathrm{p}<0.01$ and $\mathrm{r}=0.625, \mathrm{p}<0.01$ respectively). Soil $\mathrm{pH}$ was positively correlated with soil moisture $(\mathrm{r}=0.522, \mathrm{p}<0.01)$ but negatively correlated with soil organic carbon and soil organic matter $(r=-0.335, p<0.05$ for both the cases).

A total of six species of crabs were recorded from the two study sites. These included three ocypodid species [Uca rosea (Tweedie, 1937), Uca triangularis (A. Milne-Edwards, 1873) and Uca dussumieri (H. MilneEdwards, 1852)] and one species from each sesarmid [Episesarma mederi (A. Milne-Edwards, 1854)], portunid [Scylla serrata (Forskål, 1775)], menippid [Myomenippe hardwickii (Gray, 1831)]. Out of the three ocypodid species, U. triangularis was present only at site 1 , whereas $U$. rosea was found to be the most dominant species at both the sites. When an independent sample t-test was performed between the two sites, they differed significantly in terms of species evenness $(\mathrm{J})$ and dominance (D) indices accordingly $(\mathrm{p}<0.05)$.

Species density was found to be much lower at site 2 (27-32 ind. $\left.\mathrm{m}^{-2}\right)$ than site 1 (58-68 ind. $\left.\mathrm{m}^{-2}\right)$. Site 1 showed a seasonal pattern in diversity indices. The premonsoon season represented significantly higher species evenness $(J)(p=0.000)$ than the post-monsoon and monsoon seasons (Fig. 2), whereas at site 2, J and D were found to be significantly different $(p=0.000)$ among three respective seasons. Throughout the study period post-monsoon was found to be the most favourable season for abundance and even distribution of crabs.

One-way ANOSIM represented significant differences in brachyuran crab community structure between

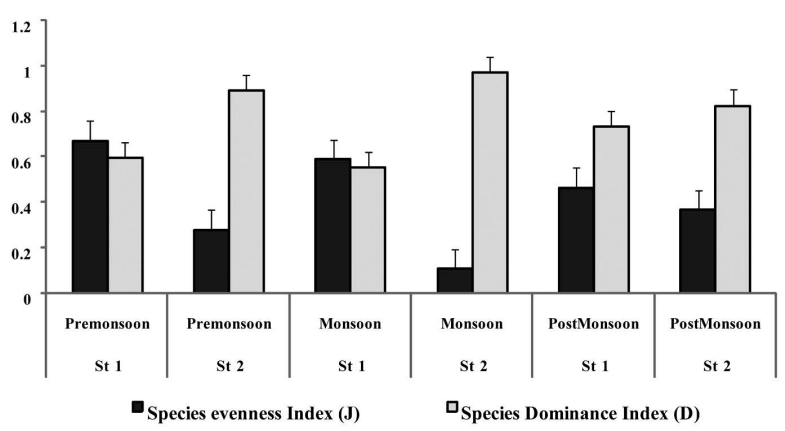

Fig. 2. - Seasonal and inter-annual variation of Pielou's evenness (J) and species dominance (D) indices for two study sites.

the two sites $(\mathrm{R}=0.05, \mathrm{p}<0.001)$. Similarity percentage analyses (SIMPER) of square-root-transformed brachyuran crab abundance data revealed that site 2 had the highest similarity $(62.6 \%)$ constituted mainly due to the contribution of only one species: $U$. rosea (cumulative contribution to abundance $62 \%$ compared with $56.6 \%$ at site 1). The discriminating species that contributed most to the dissimilarity between the two were $U$. rosea $(40.9 \%)$ and $U$. triangularis $(21.4 \%)$. The nMDS plot (Fig. 3) based on the species abundance in three seasons in three years at both study sites represented a clear division of the crab community into five different groups (derived from $60 \%$ of Bray-Curtis similarities; stress value, 0.02). The first group was composed of the abundance of $U$. rosea at both sites; the second was formed by the abundance of Myomenippe hardwicki (Gray, 1831) at site 1; the third group was formed by the absence of $U$. triangularis at site 2 ; and the fourth group was mainly formed by the cumulative abundance of Uca dussumieri (H. Milne-Edwards, 1852) and $M$. hardwicki at site 2 . The fifth group was formed by all the other remaining species at both sites.

With regard to the percentage abundance of individuals, one-way ANOVA showed significant differences $(p<0.05)$ between seasons in each year and between 


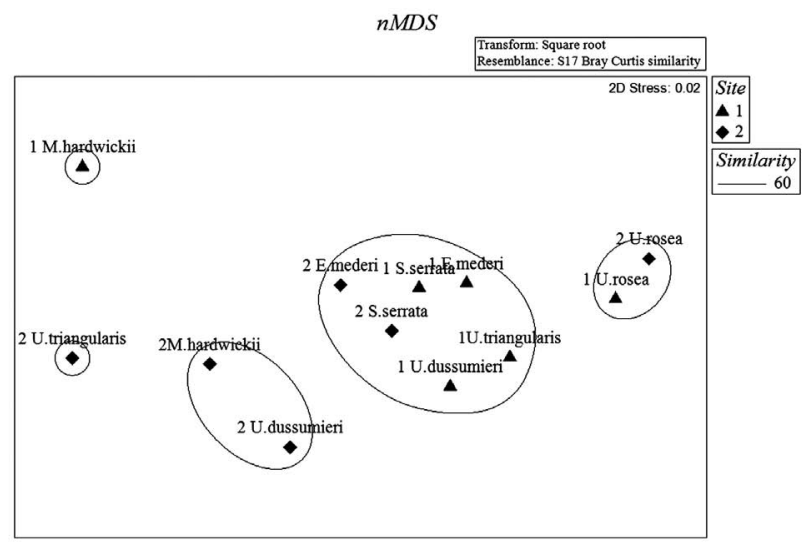

Fig. 3. - Two-dimensional nMDS plot of the crab assemblages (based on crab abundances) according to Bray-Curtis similarity. Stress value (2D): 0.02 .

years for each season at two study sites, as shown in Figure 4. When seasons were compared, $U$. rosea was found to be the highest in percentage of individuals representing maximum abundance during all seasons at both sites. A significantly higher percentage abundance of $U$. triangularis was recorded in the pre-monsoon season than in the other two seasons in every year at site 1; E. mederi was also comparatively higher in percentage during the monsoon than in the other two seasons. At site 2, U. rosea was the most dominant species throughout the year, followed by $S$. serrata and E. mederi.

Figure 5 shows the CCA analysis for the brachyuran crab assemblage based on 36 samples at each of the stations, including all the seasons in the study period. It indicates that the environmental variables explain significantly about $96 \%$ and $97.5 \%$ of the crab assemblages at site 1 and site 2, respectively, considering a cumulative percentage variance of four axes. The brachyuran crab species at each site are plotted as the points in relation to the environmental parameters

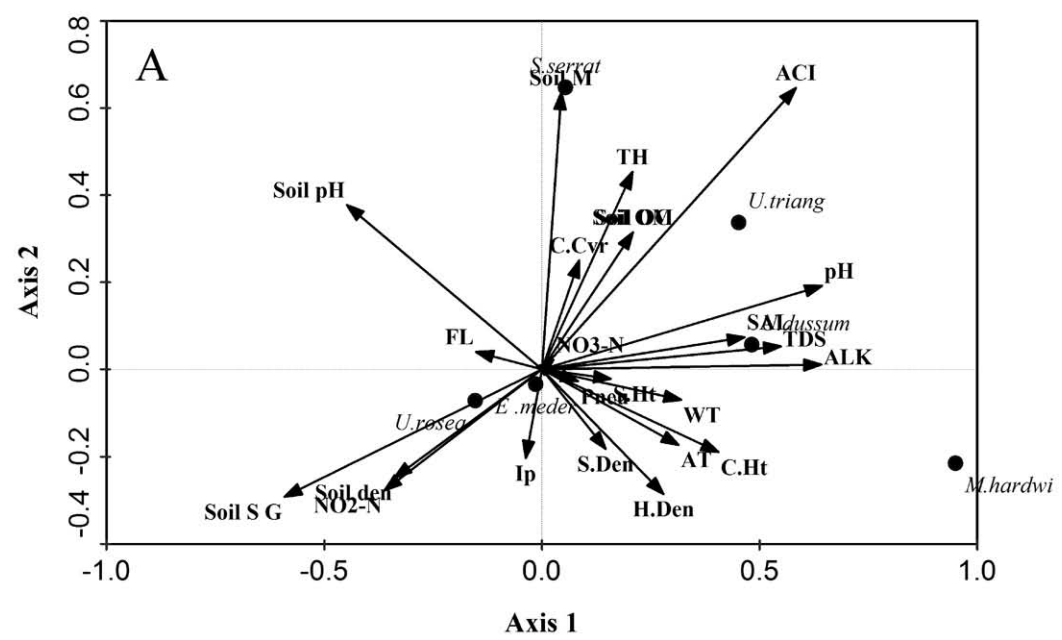

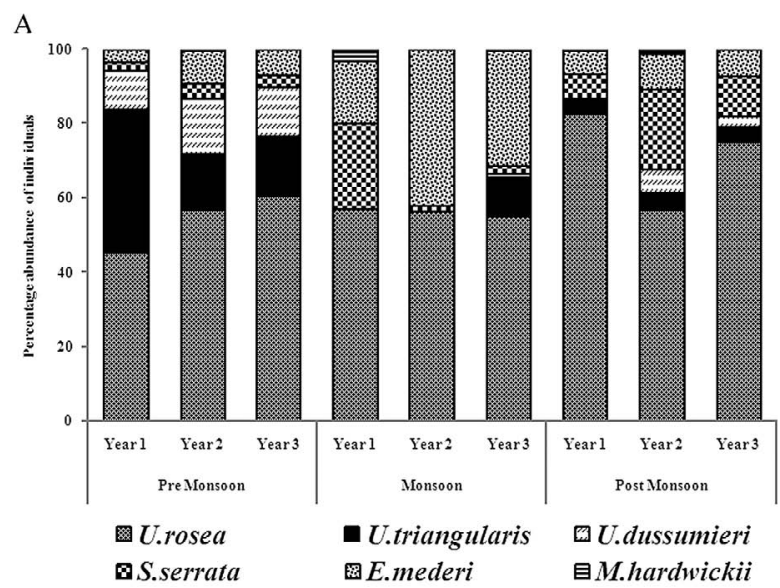

B

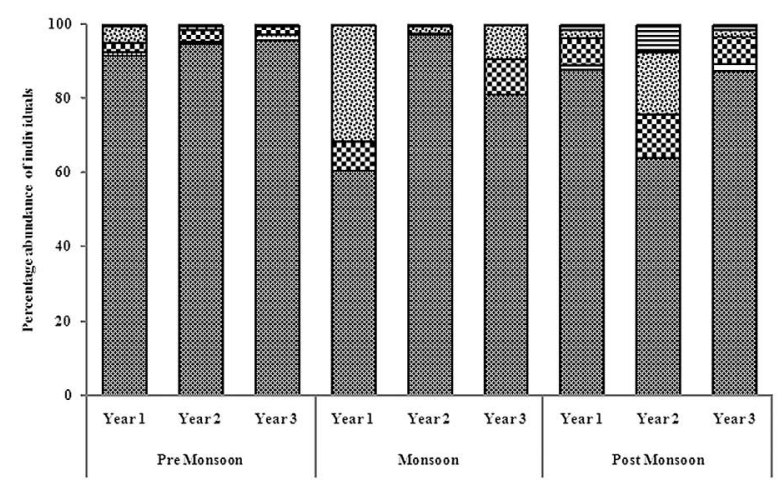

Fig. 4. - Seasonal and interannual species composition at site 1 (A) and Site 2 (B).

plotted as arrows. Although a number of axes were determined within the study, only axes 1 and 2 were taken into consideration as they accounted for $77.5 \%$ and $75.2 \%$ of the total variability of the four axes.

Considering their vector length, acidity $(\mathrm{r}=0.58)$, $\mathrm{pH}$ of water $(\mathrm{r}=0.64)$, alkalinity $(\mathrm{r}=0.64)$, TDS $(\mathrm{r}=0.55)$

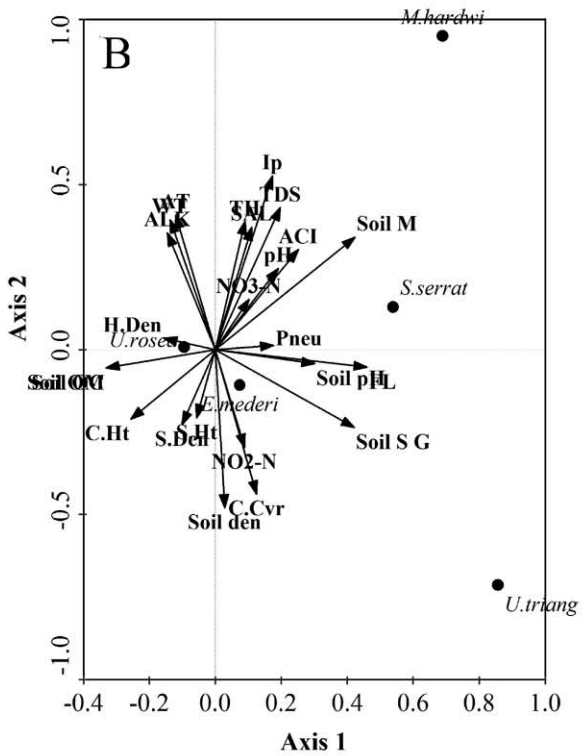

Fig. 5. - The CCA Graph showing species vs. environmental correlation at site 1 (A) and site 2 (B). Abbreviations: U. rosea, Uca rosea; U. triang, Uca triangularis; U. dussum, Uca dussumieri; E. meder, Episesarma mederi; M. hardwi, Myomenippi hardwicki; S. serrat, Scylla serrata; C.Cvr, canopy cover; C. Ht, canopy height; S. Den, shrub density; S. Ht, shrub height; Pneu, pneumatophore density; H. Den, herb density; FL, fallen leaves; Soil OC, soil organic carbon; Soil OM, soil organic matter; Soil M, soil moisture; Soil den, soil density; Soil S G, soil specific gravity; WT, water temperature; AT, air temperature; ALK, alkalinity; ACI, Acidity; TH, total hardness; SAL, Salinity; Ip, inorganic phosphate; NO3-N, nitrate-nitrogen; NO2-N, nitrite-nitrogen. 
and soil specific gravity $(\mathrm{r}=-0.59)$ might be the most important environmental parameters influencing the crab assemblage in site 1(Fig. 5A). On the other hand, inorganic phosphate content of water $(\mathrm{r}=0.51)$, soil moisture $(\mathrm{r}=0.42)$, soil density $(\mathrm{r}=-0.46)$, soil specific gravity $(\mathrm{r}=0.42)$ and canopy cover $(\mathrm{r}=-0.42)$ were the important environmental variables that influenced the crab assemblage at site 2 (Fig. 5B). At site 1, axis 1 separated the $\mathrm{pH}$-sensitive, acidity-tolerant species (on the right) from the species preferring a nutrient-rich environment. Axis 1 also explained $S$. serrata to be sensitive to changes in the moisture content of soil. Again, both axes separated the species with highest acidity tolerance (the upper half) and lower acidity tolerance (the lower half). At site 2, axis 1 separated the species which preferred heavier soil (contributing to the higher values of soil density and soil specific gravity) than the others. Axis 2 separated mostly the species having affinity towards inorganic phosphate (the upper half) from the species sensitive to it (the lower half).

\section{DISCUSSION}

Among all the benthic macro fauna which dwells in the intertidal zone, brachyurans are the most prominent (Jones 1984, Macintosh 1988) as they play a fundamental role in the structuring and functioning of the mangrove ecosystem (Lee 1999). Although extensive literature exists on crab diversity in southeast Asia and even in the coastal region of India (Tan and $\mathrm{Ng} 1994$, Ashton et al. 2003b, Sakthivel and Fernando 2012), very few (Chakraborty and Choudhury 1992a,b) have dealt recently with crab diversity in Sundarban.

The number of crab fauna recorded in different climatic zones of the world varies considerably. Ashton et al. (2003a) have recorded 31 species of crab from Sarawak mangrove in Indonesia. The number of species found during the present study is low compared with other previous studies from the country (Table 3). However, the crab diversity in Sundarban has not been studied extensively. Moreover, the effect of habitat on the crab diversity has not yet been explored in this mangrove. The present study establishes that the brachyuran crab fauna reflects habitat status when natural mangrove forests and replanted forests are compared. Within the limitations of the sampling, two interesting facts appear to affect the brachyuran crab community structure most strongly: the age of the forest stand and the level of anthropogenic turbulence.
Six species of brachyuran crabs were recorded from two different study sites. Among the two, all the species were abundant at site 1 in all seasons. The higher values of D at site 2 (Fig. 2) can be directly attributed to the maximum predominance of a particular species, $U$. rosea. It seems from the present study that most of the crab species preferred forested mudflats over replanted site. The assemblage groups revealed from the nMDS analysis (Fig. 3) showed clear separation according to species abundance. The high dominance of $U$. rosea, widely distributed at the replanted site and in the forested mangrove, contributed the overall lower diversity values. Absence of $U$. triangularis at the replanted site and low abundance of $M$. hardwicki placed them in the separate plots from the others. At site 2, microhabitat differentiation was not prominent due to low canopy cover (up to 20\%) and root ramification. Hence, absence of $U$. triangularis, a spatially inferior competitor, is a foregone conclusion due to presence of all other competitively superior species.

Tan and Ng (1994) suggested that the maintenance of high crab species diversity is integral to the health of the mangroves. Natural and human-induced disturbances pose serious threats to the functioning of mangrove ecosystems (Osborn and Polsenberg 1996). The reaction of natural ecosystem to disturbances is often discontinuous, determined inadvertently and hence unpredictable (Holling et al. 1995). The present study shows that brachyuran crab community structure changes with the age of the stand. High ocypodid crab abundance dominates disturbed and young plantations (site 2), whereas a large abundance of grapsoid crabs, exclusively sesarmids, dominates mature forests where the canopy closes. Similar observations have been established elsewhere in different mangroves of the world (Macintosh 1984, Aksornkoae 1993, Sasekumar and Chong 1998, Ashton et al. 2003b). The result of independent sample t-test of the two study sites varied significantly $(\mathrm{p}<0.05)$ in terms of pneumatophore density, herb density, canopy cover and number of decaying leaves. Site 2 has a lesser number of Avicennia sp. facing the river, resulting in a lesser number of breathing roots. Reduction in the pneumatophore density and the attached algal mass would lead to higher near-bottom flow rates, with a concomitant reduction in deposition of nutrients (Abelson and Denny 1997). The present study establishes $U$. rosea to be an opportunistic species which has a negative correlation with the pneumatophore density and therefore a lesser need

Table 3. - Previous reported works on mangrove crabs in India.

\begin{tabular}{|c|c|c|}
\hline Geographical Location & Authors & $\begin{array}{l}\text { No. of brachyuran } \\
\text { species reported }\end{array}$ \\
\hline Sagar island $\left(21^{\circ} 31^{\prime} \mathrm{N}\right.$ to $21^{\circ} 53^{\prime} \mathrm{N}$ and $88^{\circ} 02^{\prime} \mathrm{E}$ to $\left.88^{\circ} 15^{\prime} \mathrm{E}\right)$ & Chakraborty et al. 1992 & 14 \\
\hline Prentice Island $\left(21^{\circ} 40^{\prime} \mathrm{N}\right.$ to $21^{\circ} 48^{\prime} \mathrm{N}$ and $88^{\circ} 16^{\prime} \mathrm{E}$ to $\left.88^{\circ} 21^{\prime} \mathrm{E}\right)$ & Chakraborty and Chaudhury $1992 \mathrm{a}$ & 18 \\
\hline Sagar island $\left(21^{\circ} 31^{\prime} \mathrm{N}\right.$ to $21^{\circ} 53^{\prime} \mathrm{N}$ and $88^{\circ} 02^{\prime} \mathrm{E}$ to $\left.88^{\circ} 15^{\prime} \mathrm{E}\right)$ & Chakraborty and Chaudhury 1992 b & 4 \\
\hline Pichavaram Mangrove & Ravichandran et al. 2000 & 23 \\
\hline Pichavaram Mangrove $\left(11^{\circ} 27^{\prime} \mathrm{N}\right.$ and $\left.79^{\circ} 47^{\prime} \mathrm{E}\right)$ & & 38 \\
\hline Vellar Mangrove $\left(11^{\circ} 29^{\prime} \mathrm{N}\right.$ to $\left.79^{\circ} 46^{\prime} \mathrm{E}\right)$ & Ajmal Khan et al. 2005 & 8 \\
\hline Pichavaram Mangrove $\left(11^{\circ} 27^{\prime} \mathrm{N}\right.$ and $\left.79^{\circ} 46^{\prime} \mathrm{E}\right)$ & Ravichandran et al. 2007 & 46 \\
\hline Karawar mangrove, west coast of India $\left(74^{\circ} 18^{\prime} \mathrm{N}\right.$ and $\left.14^{\circ} 88^{\prime} \mathrm{E}\right)$ & Bandekar et al. 2011 & 13 \\
\hline Gulf of Kutch & Trivedi et al. 2012 & 19 \\
\hline Mudasal Odai $\left(11^{\circ} 29^{\prime} \mathrm{N}\right.$ and $\left.79^{\circ} 47^{\prime} \mathrm{E}\right)$ and Nagapattim $\left(10^{\circ} 46^{\prime} \mathrm{N}\right.$ and79 $\left.59^{\prime} \mathrm{E}\right)$ & Sakthivel and Fernando 2012 & 38 \\
\hline
\end{tabular}


for deposited nutrients, a finding which is corroborated by Macintosh (1984). Open mudflats provide a unique habitat for many species of brachyuran crabs; 10 species of brachyuran crabs have been recorded from the open mudflat of Mahi river estuary (Pandya and Vachhrajani 2013). However, in the current study, out of the six species of crab recorded, $U$. rosea dominates the replanted open mudflat habitat, whereas $U$. triangularis mostly prefers forested mangrove.

Elucidating the relationship between species occurrence and environmental variables allows us to identify the ecological processes that regulate different crab populations. Regardless of the changes in environmental conditions, many species change their habitats according to their ontogeny or seasonal rhythms, which means that relations between different species and their environment or habitat are spatially and seasonally dynamic (Morrison et al. 2002, Kanou et al. 2005). Mangrove crabs are generally affected by the abiotic and biotic factors of the habitat (Macintosh 1988, Lee 1999, Ashton et al. 2003b). The CCA in the present investigation recognized that hydrological and edaphic factors and the physical architecture of the habitat have a great impact on the brachyuran assemblage. The findings were similar to earlier observations (Soundarapandian et al. 2008, Chakraborty and Choudhury 1992b). The crab assemblage of the study area of Sundarban mangrove is influenced seasonally by a variety of factors, viz. acidity, $\mathrm{pH}$ content of water, alkalinity, soil moisture content, soil $\mathrm{pH}$, nutrient content of water and habitat attributes such as canopy cover and canopy height.

High dominance of a single species might indicate a stressful environment (Macintosh et al. 2002 and Ashton et al. 2003b). As the ecosystem has not been restored to the normal mature condition by replantation, only one species ( $U$. rosea) dominates the crab fauna at site 2. Further time may change the crab community structure at that location or the anthropogenic effects may irreversibly prevent the ecosystem from going back to its original state (Ashton et al. 2003b). The crab community composition may give an indication of the habitat health. Ghost crabs have already been used as a tool for rapid assessment of human impacts on the exposed sandy beaches (Barros 2001). In mangrove, given the environmental complexity, it is uncertain whether one or two species could be sufficiently sensitive to all major factors affecting a complex ecosystem to serve as indicator species. Rather, monitoring the changes in community structure may provide a more prominent and effective tool for evaluating ecosystem health (Cairns et al. 1993 and Ashton et al. 2003b). As the brachyuran crab communities are omnipresent throughout the Sundarban mangrove and are an important part of the mangrove food web, they can be used as a good indicator of the local condition. The fact that they are primarily sedentary and have a reasonably long life cycle (Ashton et al. 2003b) also supports their candidature. Changes in the crab community thus reflect the mangrove habitat status, which can be used as a tool for management purposes.

In conclusion, the determinant role of the habitat characteristics in moderating crab abundance and di- versity is indicated. More meticulous approaches to the assessment of past and current restoration efforts must be developed (Stevenson et al. 1999) in accordance with the study of the health of the mangrove at undisturbed natural sites. An understanding of the ecological effects of disturbance to mangrove forests and the changes in the structure and dynamics of the crab meta-community will be of great importance for conservation of this unique ecosystem.

\section{ACKNOWLEDGEMENTS}

The authors are thankful to the local villagers of Sundarbans, and to the Head of the Department of Zoology, University of Calcutta, India for the facilities provided. Financial support from the University Grant Commission-National Eligibility Test project is gratefully acknowledged.

\section{REFERENCES}

Abelson A., Denny M. 1997. Settlement of marine organisms in flow. Annu. Rev. Ecol. Syst. 28: 317-339. http://dx.doi.org/10.1146/annurev.ecolsys.28.1.317

Acosta C.A., Butler M.J. 1997. Role of mangrove habitat as a nursery for juvenile spiny lobster, Panulirus argus, in Belize. Mar. Freshw. Res. 48(8): 721-728. http://dx.doi.org/10.1071/MF96105

Ajmal Khan S., Rafii S.M., Lyla P.S. 2005. Brachyuran crab diversity in natural (Pitchavaram) and artificially developed mangroves (Vellar estuary). Current Sci. 88(8): 1316-1324.

Aksornkoae S. 1993. Ecology and management of mangroves. IUCN, AIT, Bangkok, Thailand.

APHA. 1998. Standard methods for the examination of water and wastewater, 20th ed. Washington, DC, $1325 \mathrm{pp}$.

Ashton E.C., Macintosh D.J., Hogarth P.J. 2003a. A baseline study of the diversity and community ecology of crab and molluscan macrofauna in the Sematan mangrove forest, Sarawak, Malaysia. J. Trop. Ecol. 19: 127-142. http://dx.doi.org/10.1017/S0266467403003158

Ashton E.C., Hogarth P.J, Macintosh D.J. 2003b. A comparison of brachyuran crab community structure at four mangrove locations under different management systems along the Melaka Straits-Andaman Sea Coast of Malaysia and Thailand. Estuaries 26(6): 1461-1471 http://dx.doi.org/10.1007/BF02803654

Bandekar P.D., Neelkantan K., Kakati V.S. 2011. Biodiversity of crabs in Karawar mangrove environment West Coast of India. Recent Res. Sci. Technol. 3(4): 1-5.

Barros F. 2001. Ghost crabs as a tool for rapid assessment of human impacts on exposed sandy beaches. Biol. Conserv. 97: 399-404. http://dx.doi.org/10.1016/S0006-3207(00)00116-6

Berger W.H., Parker F.L. 1970. Diversity of planktonic foraminifera in deep sea sediments. Science 168: 1345-1347. http://dx.doi.org/10.1126/science.168.3937.1345

Brower J.E., Zar J.H., von Ende C.N. 1998. Field and laboratory methods for general ecology, 4th edn., McGraw-Hill, New York.

Cairns J., Mccormick P.V., Niederlehner B.R. 1993. A proposed framework for developing indicators of ecosystem health. Hydrobiol. 263: 1-44. http://dx.doi.org/10.1007/BF00006084

Chakraborty S.K., Choudhury A. 1992a. Ecological studies on the zonation of brachyuran crabs in a virgin mangrove island of Sundarbans, India. J. Mar. Biol. Ass. India 34(1-2): 189-194.

Chakraborty S.K., Choudhury A. 1992b. Population Ecology of Fiddler Crabs (Uca spp.) of the mangrove estuarine complex of Sundarbans, Trop. Ecol. 33(1): 78-88.

Chakraborty S.K., Poddar T.K., Choudhury A. 1992. Species diversity of macrobenthos of Sagar Island, Sundarbans, India. Proc. Zool. Soc. Calcutta 45(Suppl. A): 435-444.

Clarke K.R. 1993. Non-parametric multivariate analyses of changes in community structure. Aust. J. Ecol. 18: 117-143. http://dx.doi.org/10.1111/j.1442-9993.1993.tb00438.x 
Clarke K.R., Warwick R.M. 1994. Changes in marine communities: An approach to statistical analysis and interpretation. Plymouth Marine Laboratory, Plymouth, U.K.

Ellner S.P. 2001. Pair approximation for lattice models with multiple interaction scales. J. Theor. Biol. 210: 435-347 http://dx.doi.org/10.1006/jtbi.2001.2322

Golley F., Odum H.T., Wilson R.L. 1962. The structure and metabolism of Puertorican and mangrove forest in May. Ecology 43: 9-18. http://dx.doi.org/10.2307/1932034

Gopal B., Chauhan M. 2006. Biodiversity and its conservation in the Sundarban Mangrove Ecosystem. Aquat. Sci. 68: 338-354. http://dx.doi.org/10.1007/s00027-006-0868-8

Grasshoff K. 1983. Determination of nitrite, nitrate, oxygen, thiosulphate. In: Grasshoff K., Ehrhardt M., Kremling K. (eds), Methods of seawater analysis. Verlag Chemie Weinheim, New York, pp. 61-150.

Grasshoff K., Ehrhardt M., Kremling K. (eds) 1983. Methods of seawater analysis. Verlag Chemie Weinheim, New York, 419 pp.

Hatcher B.G., Johannes R.E., Robertson, A.I. 1989. Review of research relevant to the conservation of shallow tropical marine ecosystems. Oceanogr. Mar. Biol. Annu. Rev. 27: 337-414.

Holling C.S., Schindler D., Walker W., et al. 1995. Biodiversity in the functioning of ecosystems: An ecological synthesis. In: Perrings C., Maler K.G., Folke C., et al. (eds), Biodiversity Loss: Economic and Ecological Issues. Cambridge University Press, Cambridge, U.K., pp. 44-83. http://dx.doi.org/10.1017/CBO9781139174329.005

Jones D. A. 1984. Crabs of the mangal ecosystem. In: Por F.D., Dor I. (eds), Hydrobiology of the Mangal. W. Junk Publishers, The Hague, Netherlands, pp. 89-109.

Jongman R.H.G., Ter Braak C.J.F., Van Tongeren O.F.M. 1995. Data analysis in community and landscape ecology. Cambridge University Press, Cambridge. http://dx.doi.org/10.1017/CBO9780511525575

Kanou K., Sano M., Kohno H. 2005. Larval and juvenile fishes occurring with flood tides on an intertidal mudflat in the Tama River estuary, central Japan. Ichthyol. Res. 52: 158-164. http://dx.doi.org/10.1007/s10228-005-0267-5

Kathiresan K., Bingham B.L. 2001. Biology of mangroves and mangrove ecosystems. Adv. Mar. Biol. 40: 81-251. http://dx.doi.org/10.1016/S0065-2881(01)40003-4

Kon K., Kurokura H., Tongnunui P. 2009. Do mangrove root structures function to shelter benthic macrofauna from predators? J. Exp. Mar. Biol. Ecol. 370(1-2): 1-8. http://dx.doi.org/10.1016/j.jembe.2008.11.001

Laegdsgaard P., Johnson C. 2001. Why do juvenile fish utilise mangrove habitats? J Exp. Mar. Biol. Ecol. 257(2): 229-253. http://dx.doi.org/10.1016/S0022-0981(00)00331-2

Lawton J. H. 1983. Plant architecture and the diversity of phytophagous insects. Annu. Rev. Entomol. 28: 23-39. http://dx.doi.org/10.1146/annurev.en.28.010183.000323

Lee S. Y. 1998. Ecological role of grapsid crabs in mangrove ecosystems: A review. Mar. Freshw. Res. 49: 335-343. http://dx.doi.org/10.1071/MF97179

Lee S.Y. 1999. Tropical mangrove ecology: Physical and biotic factors influencing ecosystem structure and function. Aust. J. Ecol. 24: 355-366. http://dx.doi.org/10.1046/j.1442-9993.1999.00984.x

Levings S.C., Garrity S.D., Burns K.A. 1994. The Galeta oil spill. III. Chronic reoiling, long-term toxicity of hydrocarbon residues and effects on epibiota in the mangrove fringe. Est. Coast. Shelf Sci. 38: 365-395. http://dx.doi.org/10.1006/ecss.1994.1026

Macarthur R.H., Macarthur J.W. 1961. On bird species diversity. Ecology 42: 594-598. http://dx.doi.org/10.2307/1932254

Macia A., Quincardete I., Paula, J. 2001. A comparison of alternative methods for estimating population density of the fiddler crab Uca annulipes at Saco Mangrove, Inhaca Island (Mozambique). Hydrobiol. 449: 213-219. http://dx.doi.org/10.1023/A:1017502817087

Macintosh D.J. 1984. Ecology and productivity of Malaysian mangrove crab populations (Decapoda: Brachyura). In: Soepadmo E., Rao A.N., Macintosh D.J. (eds), Proceedings of the Asian Symposium on Mangrove Environment-Research and Management. University of Malaysia, KualaLumpur, Malaysia, and UNESCO, Paris, France, pp. 354-377.

Macintosh D.J. 1988. The ecology and physiology of decapods of mangrove swamps. Symp. Zool. Soc. London 59: 315-341.

Macintosh D.J., Ashton E.C., Havanon S. 2002. Mangrove rehabilitation and intertidal biodiversity: A study in the Ranong mangrove ecosystem, Thailand. Est. Coast. Shelf Sci. 55: 331-345. http://dx.doi.org/10.1006/ecss.2001.0896

Manna S., Chaudhuri K., Bhattacharyya S., et al. 2010. Dynamics of Sundarban estuarine ecosystem: eutrophication induced threat to mangroves. Saline Systems 6: 8 http://dx.doi.org/10.1186/1746-1448-6-8

Morrison M.A., Francis M.P., Hartill B.W., et al. 2002. Diurnal and tidal variation in the abundance of the fish fauna of temperate tidal mudflat. Est. Coast. Shelf Sci. 54: 793-807. http://dx.doi.org/10.1006/ecss.2001.0857

Nelson D.W., Sommers L.E. 1996. Total carbon, organic carbon, and organic matter. In: A.L. Page et al. (eds), Methods of Soil Analysis, Part 3, Chemical Methods. Soil Science Society of America, American Society of Agronomy. pp. 961-1010. http://dx.doi.org/10.2136/sssabookser5.3.c34

Ng P.K.L., Guinot D., Davie P.J.F. 2008. Systema Brachyurorum: Part I. An Annotated Checklist of Extant Brachyuran Crabs of the World. Raffles B Zool. 17: 1-286.

Norušis M. 2000. SPSS 10.0, guide to data analysis. Upper Saddle River: Prentice-Hall.

Osborn J.G., Polsenberg J.F. 1996. Meeting of the mangrovellers: The interface of biodiversity and ecosystem function. Trends Ecol. Evol. 11: 354-356. http://dx.doi.org/10.1016/0169-5347(96)30035-9

Pandya P.J., Vachhrajani, K.D. 2013. Brachyuran crab diversity of lower estuarine mud flats of Mahi River with new record of two species from Gujarat, India. Arthropods 2(4):242-250.

Pielou E.C. 1969. An introduction to mathematical ecology. WileyInterscience, New York. $\mathrm{x}+294$ pp.

Primavera J.H. 1997. Fish predation on mangrove-associated penaeids: The role of structures and substrate. J. Exp. Mar. Biol. Ecol. 215: 205-216. http://dx.doi.org/10.1016/S0022-0981(97)00046-4

Ravichandran S., Soundarapandian P., Kannupandi T. 2000. Zonation and distribution of crabs in Pichavaram mangrove swamp, southeast Coast of India. Indian J. Fish. 47: 73-78.

Ravichandran S., Anthonisamy S., Kannupandi T., et al. 2007. Habitat Preference of crabs in Pichavaram Mangrove Environment, Southeast Coast of India. J. Fish. Aquat. Sci. 2(1):47-55. http://dx.doi.org/10.3923/jfas.2007.47.55

Robertson A. I. 1986. Leaf-burying crabs: Their influence on energy flow and export from mixed mangrove forests (Rhizophora spp.) in northeastern Australia. J. Exp. Mar. Biol. Ecol. 102: 237-248 http://dx.doi.org/10.1016/0022-0981(86)90179-6

Robertson A.I., Blaber S.J.M. 1992. Plankton epibenthos and fish communities. In: Robertson A.I., Alongi D. (eds), Tropical Mangrove Ecosystems, Springer-Verlag, New York. pp. 173-224.

http://www.agu.org/books/ce/v041/CE041p0173/CE041p0173. pdf

Sakthivel K., Fernando A. 2012. Brachyuran crabs diversity in Mudasal Odai and Nagapattinam coast of south east India. Arthropods 1(4): 136-143.

Sasekumar A., Chong V. C. 1998. Faunal diversity in Malaysian mangroves. Global Ecol. Biogeogr. 7: 57-60. http://dx doi.org/10.2307/2997697

Smith III T.J., Boto K.G., Frusher S.D., et al.1991. Keystone species and mangrove forest dynamics: The influence of burrowing crabs on soil nutrient status and forest productivity. Est. Coast. Shelf Sci. 33:419-432. http://dx.doi.org/10.1016/0272-7714(91)90081-L

Soundarapandian P., John Samuel N., Ravichandran S., et al. 2008. Biodiversity of Crabs in Pichavaram Mangrove Environment, South East Coast of India. Int J of Zool Res 4: 113-118. http://dx.doi.org/10.3923/ijzr.2008.113.118

Stevenson N.J., Lewis R.R., Burbridge P.R. 1999. Disused shrimp ponds and mangrove rehabilitation. In: Streever W. (ed.), An International Perspective on Wetland Rehabilitation. Kluwer Academic Publishers, Dordrecht, Netherlands, pp. 277-297. http://dx.doi.org/10.1007/978-94-011-4683-8_28

Strickland J.D.H., Parsons T.R. 1972. A practical handbook of seawater analysis. J. Fish. Res. Board Can.167: 1-311.

Tan C.G.S., Ng P.K.L. 1994. An annotated checklist of mangrove brachyuran crabs from Malaysia and Singapore. Hydrobiol. 285:75-84. http://dx.doi.org/10.1007/BF00005655 
Ter Braak C.J.F. 1986. Canonical correspondence analysis: A new eigenvector technique for multivariate direct gradient analysis. Ecology 67: 1167-1179.

http://dx.doi.org/10.2307/1938672

Ter Braak C.J.F. 1988. CANOCO - A Fortran program for canonical community ordination by (partial) (detrended) (canonical) correspondence analysis, principal component analysis and redundancy analysis (version 2.1). Technical Report No. LWA- 88-02.TNCO. Institute of Applied Computer Science, Statistical Department Wageningen, 6700 AC Wageningen, The Netherlands, $95 \mathrm{pp}$.

Trivedi J.N., Gadhavi M.B., Vachhrajani K.D. 2012. Diversity and habitat preference of brachyuran crabs in Gulf of Kutch, Gujarat, India. Arthropods 1(1): 13-23. 